\title{
Efficacy of titanium dioxide compounds in preventing environmental contamination by meticillin resistant Staphylococcus aureus (MRSA)
}

\author{
Chow Wai Leng ${ }^{1}$, Tin Aung Soe ${ }^{2}$, Lim Woan Wui ${ }^{3}$, Jeremy Lim Fung Yen ${ }^{4}$, \\ Asok Kurup ${ }^{5}$, Ling Moi Lin ${ }^{6}$, Tan Ai Ling ${ }^{7}$, Ong Biauw $\mathrm{Chi}^{8}$ \\ 1. Health Services Research, Eastern Health Alliance, Singapore \\ 2. Health Services Research, Eastern Health Alliance, Singapore
}

3. Trauma Service, Department of General Surgery, Khoo Teck Puat Hospital, Singapore

4. Insights Health Associates, Singapore

5. Department of Infectious Diseases, Singapore General Hospital, Singapore

6. Infection Control, Singapore General Hospital, Singapore

7. Department of Pathology, Singapore General Hospital, Singapore

8. Department of Anaesthesiology, Singapore General Hospital, Singapore

doi: 10.3396/IJIC.v9i3.022.13

\begin{abstract}
This study examined the efficacy of photocatalytic titanium dioxide $\left(\mathrm{TiO}_{2}\right)$ coating in reducing environmental MRSA contamination via a cross-sectional observational study in a tertiary hospital. This involved using environmental samplings of $\mathrm{TiO}_{2}$ treated and $\mathrm{TiO}_{2}$ untreated surfaces from single rooms in intensive care unit, open-planned intermediate care area and general ward. Planned scheduled sampling occurred up to 24 months post $\mathrm{TiO}_{2}$ treatment. Ad hoc sampling of MRSA exposed environment occurred whenever MRSA infected or colonized patient was admitted for $>48$ hours. Efficacy of $\mathrm{TiO}_{2}$ in preventing environmental contamination was computed. Culture positive rates were compared between treated and untreated surfaces, and planned and ad hoc sampling. 698 samples were obtained. Samples from untreated surfaces and ad hoc samples were more likely to be culture positive (for MRSA and other bacteria) [untreated versus treated surfaces: odds ratio (OR) 2.95, 95\% confidence interval (Cl) $1.25-6.94, \mathrm{p}=0.01$; ad hoc sampling post MRSA exposure versus planned sampling: OR 4.52, 95\% Cl $2.131-9.615, \mathrm{p}<0.001)]$. Multivariate analysis suggests only MRSA exposure influenced positive cultures. $\mathrm{TiO}_{2}$ did not influence positive culture results. More research is needed to evaluate the relative lack of $\mathrm{TiO}_{2}$ efficacy in preventing contamination.
\end{abstract}

Key words: Meticillin-Resistant Staphylococcus aureus and drug effect; Titanium Dioxide; Surface-active agents

\section{Corresponding Author}

Dr Chow Wai Leng, Assistant Director

Health Services Research, Eastern Health Alliance, Singapore

5 Tampines Central 1, Tampines Plaza, \#08-01/05, Singapore 529541

Email: wailengc@gmail.com 


\section{Introduction}

The prevalence of hospital acquired meticillin-resistant Staphylococcus aureus (MRSA) infections has not declined in the United States ${ }^{1}$, in parts of Europe ${ }^{2,3}$ and in Singapore ${ }^{4}$ where MRSA accounts for more than $25 \%$ of Staphylococcus aureus isolates. An increasing trend of MRSA has been documented in the United States from 1998 - 2007. ${ }^{5}$ In 2002, MRSA infections was estimated to cost the United States $\$ 35.7$ billion to $\$ 45$ billion annually. ${ }^{6}$ In the United Kingdom, this was more than $£ 45$ million annually. ${ }^{7}$

Evidence suggests that environmental contamination plays an important role in patient acquisition of hospital acquired infections (HAI), ${ }^{8,9,10}$ This is especially so for MRSA which has been demonstrated to contaminate many hospital items such as lockers, overbed tables and beds ${ }^{11,12}$ and can remain viable on dry surfaces for months. ${ }^{13}$

Photocatalytic titanium dioxide $\left(\mathrm{TiO}_{2}\right)$ substrates eliminate organic compounds and act as a disinfectant upon activation via illumination with visible-light. They continue to have extended anti-bactericidal capabilities after application to surfaces via spray painting technique. A 'binder' which is subject to wear and tear, acts as a glue to adhere the $\mathrm{TiO}_{2}$ to the surface (Information as provided by the distributor). Such $\mathrm{TiO}_{2}$ compounds can therefore be potentially utilized as an adjunct to current cleaning techniques.

Despite their potential use in the healthcare setting, there are no studies to date, to the best of our knowledge, which evaluate the efficacy of $\mathrm{TiO}_{2}$ in the real healthcare environment.

We therefore sought to examine the efficacy of $\mathrm{TiO}_{2}$ disinfectant as an adjunct to conventional terminal cleaning in preventing environmental contamination after exposure to patients with and without MRSA. We also examined the prevalence of MRSA and multi-resistant Gram-negative bacilli (MRGNB) of nosocomial significance such as Pseudomonas aeruginosa and Acinetobacter baumannii on different surfaces in the hospital.

\section{Materials and Methods}

\section{Setting}

A cross-sectional observational study was conducted in a 1500-bedded tertiary hospital in November 2007 to May 2009. Baseline MRSA incidence rate was 0.6 infections per 1000 patient-days in 2007 and 2008, declining to 0.4 per 1000 patient-days in $2009 .^{14}$

Environmental samples were drawn from a single ward that consisted of an Intensive Care Unit (ICU) of eight single-bedded isolation rooms, an open plan fourbedded ward bay Intermediate Care Area (ICA) and a normal general ward (GW) with three six-bedded ward bays. Two ICU single bed isolation rooms and four ICA beds were treated with $\mathrm{TiO}_{2}$ in May 2007. $\mathrm{TiO}_{2}$ was applied on surfaces and fixed furniture such as the bed frames, door handles and taps.

Both MRSA and non-MRSA patients were admitted to the ward as determined by patients' clinical conditions and randomly assigned to an appropriate and available location.

\section{Environmental sampling and microbiological methods}

Environmental sampling involved: 1) sampling of equal number of treated and untreated surfaces at planned intervals of 6-, 9-, 12-, 18- and 24 months post $\mathrm{TiO}_{2}$ treatment; and 2) ad hoc sampling of surfaces after MRSA positive patients, both colonized and infected, were admitted for $>48$ hours to the ward. The surfaces sampled could either be treated or untreated. This was determined by the random bed allocation of MRSA positive patients.

$\mathrm{TiO}_{2}$ coating (EnviroCare ${ }^{\circledR}$ ) was applied according to manufacturers' instructions to two ICU rooms and the ICA. Paired scheduled sampling of treated and untreated surfaces were taken till 24 months post treatment to examine the $\mathrm{TiO}_{2}$ extended antimicrobial efficacy that was within the manufacturer's warranty of 36 months and advice to re-coat wear and tear surfaces every $18-24$ months.

Six to eight frequently touched surfaces among eleven surfaces (bedside locker - top surface and drawer, tap at basin, door pad, main light switch, haemodynamic 
monitor screen, carpenter ruler, common blood pressure cuff, pull switch for wall-mount lamp, screening curtain around bed and entrance door handle) were sampled, depending on the room type. Dacron tipped sterile swabs moistened in sterile brain heart infusion broth (BHIB) were used to sample surface areas of $5 \times 5 \mathrm{~cm}$ by standardized swabbing using a non-sterile template by trained personnel. Culture for MRSA and MRGNB was performed using enrichment (BHIB) incubated at $35^{\circ} \mathrm{C}$ overnight and then plated onto MRSA Select ${ }^{\mathrm{TM}}$ (from Bio-Rad), Blood agar plate (from BBL) and MacConkey agar plate (from Oxoid) and incubated at $35^{\circ} \mathrm{C}$ for up to 48 hours for MRSA Select ${ }^{\mathrm{TM}}$ plates, and 24 hours for Blood agar and MacConkey agar plates. Environmental sampling was not performed immediately after cleaning was completed.

MRSA Select ${ }^{\mathrm{TM}}$ plates were examined for presumptive MRSA growth and confirmed with latex agglutination (Pastorex $^{R}$ Staph Plus, from Bio-Rad). Blood and MacConkey agar plates were examined for Gramnegative bacteria of nosocomial significance e.g. Pseudomonas aeruginosa and Acinetobacter baumannii, and confirmed with usual biochemical tests. Susceptibility testing was done using disk diffusion technique on Mueller Hinton agar.

\section{Conventional terminal cleaning}

Conventional terminal cleaning is environmental cleaning conducted after discharge of an infectious patient. Horizontal surfaces including bed frames, patient's bedside locker and cabinets were sanitized with phenolic-based disinfectant (chlorophene 4.75\% and o-phenylphenol $4.75 \%$, dilution 1:128). Window and wall areas were cleaned with their respective cleaners. The sink was cleaned with a cleaner and sanitized with phenolic-based disinfectant. The floor was mopped with a quaternary ammonium compound based disinfectant (quaternary ammonium compounds, benzyl-c12-c16-alkyldimethyl, chlorides 9.5\%). Carpeted floors were vacuumed. Phenolicbased disinfectant was used if spills had occurred. The bedstead, mattress and pillow(s), over-bed table, infusion stand, bedside locker, cabinet, drawers, and cupboard were emptied of contents and cleaned with the quaternary ammonium compound based general purpose cleaning disinfectant and air-dried. Curtains were laundered at $70^{\circ} \mathrm{C}$ in a cycle of washing, drying and ironing and new curtains put up. Bins were emptied, and scrubbed both inside and outside with general purpose cleaning disinfectant before being rinsed and dried.

\section{General cleaning protocol}

Daily general cleaning consisted of cleaning of the surfaces in the vicinity of the patient. General ward cleaning for ICU and patients with multi-drug resistant organisms (MDRO) involved the use of a phenolic compound (chlorophene $4.75 \%$ and o-phenylphenol $4.75 \%$, dilution $1: 128$ ) while a quaternary ammonium compound (quaternary ammonium compounds, benzyl-c12-c16-alkyldimethyl, chlorides 9.5\%, dilution 1:128) was used for ICA and GW.

\section{The titanium dioxide product and application process}

The product under study was Envirocare ${ }^{\circledR}$, a $\mathrm{TiO}_{2}$ based photo-catalyst high transparency substance that was effective under sunlight or fluorescent light. Ultraviolet light was not required for its activation. [According to test reports by distributor in a comparison of decomposition of red ink under sunlight as compared to other $\mathrm{TiO} 2$ compounds.] Organic matter is decomposed to water and carbon dioxide after interaction with oxygen radicals upon contact with the coating. ${ }^{15,16}$

Surfaces and fixed furniture were surface cleaned with detergent before $\mathrm{TiO}_{2}$ application using high volume low pressure technique. The coating was sprayed on about 20 centimeters perpendicularly from the surface such that the diameter of the spray on the surface to be about 10 - 15 centimeters using high dense spraying equipment with a coating speed of $12-15$ meters per minute. The coating was then air dried.

\section{Statistical analysis}

We used individual environmental sample as a sampling unit. Overall prevalence of positive environmental culture was calculated. Specific prevalence of MRSA and gram negative bacteria were estimated based on: 1) sampling schedule (planned or ad hoc sampling); 2) sample site; 3) treatment with $\mathrm{TiO}_{2}$ (treated or untreated); and 4) room type (ICU, ICA or GW). 
$\mathrm{TiO}_{2}$ efficacy for prevention of environmental contamination by MRSA and other bacteria was computed using rate of positive culture in untreated room minus rate of positive culture in treated rooms divided by rate of positive culture in untreated rooms.

Bivariate analyses (Chi-square tests for proportion) were used to assess the association between prevalence rates and sample sites, sampling schedule (planned or ad hoc sampling), room types and treatment with $\mathrm{TiO}_{2}$.

Multiple logistic regression model was used to test the independent effect of study variables on samples returning culture positive. Only variables associated with positive culture results in bivariate analysis $(p<0.05)$ were included in the model. Level of significance was set at $\mathrm{p}<0.05$ for all statistical procedures. SPSS Window version 17 (Statistical Package for Social Science) was used for the analyses.

\section{Results}

Of the 698 samples, 563 (80.7\%) were from surfaces not treated with $\mathrm{TiO}_{2}$ (i.e. untreated) and 135 (19.3\%) were from surfaces treated with $\mathrm{TiO}_{2}$ (i.e. treated).

$67.2 \%$ were ad hoc samples from the surroundings of MRSA patients (i.e. MRSA environment) and 32.8\% were planned samples from non-MRSA patients (i.e. non-MRSA environment).

$48.7 \%, 42.1 \%$ and $9.2 \%$ of samples were drawn from ICU, GW and ICA respectively.

\section{Prevalence of environmental contamination}

Overall, $10.6 \%$ of samples were culture positive $[M R S A=9.2 \%$, other Gram-negative bacteria (GNB) $=1.4 \%]$. No multi-resistant Gram-negative bacilli (MRGNB) of nosocomial significance were isolated. Of the untreated surfaces sampled, $12.1 \%$ (68 of 563) versus $4.4 \%$ (6 of 135 ) of treated surfaces sampled were culture positive. Samples from untreated surfaces compared to treated surfaces were more likely to be culture positive [Odds ratio (OR) 2.95, 95\% Confidence Interval $(\mathrm{Cl}) 1.25-6.94, \mathrm{p}=0.01]$. Ad hoc samples from MRSA environment $(14.1 \%$ versus $3.5 \%$ planned samples) were also more likely to be culture positive (OR 4.52, 95\% Cl 2.131 - 9.615, $p<0.001$ ) (Table I).

Although prevalence of positive cultures differed among room types, this was not statistically significant. $13.9 \%$ (41 of 294) of GW samples, 8.8\% (30 of 340) of ICU samples and $4.7 \%$ (3 of 64 ) of ICA samples were positive respectively.

In addition, the common blood pressure cuff and screening curtain around the bed were the two most frequently culture positive sites (Table II).

\section{Efficacy of $\mathrm{TiO}_{2}$ in preventing environmental contamination}

When exposed to MRSA during ad hoc sampling, $12.1 \%$ of samples from untreated surfaces were culture positive (for MRSA and other bacteria) versus $4.4 \%$ among treated surfaces. None of the treated surfaces were positive during planned sampling (Table III).

Table I. Factors influencing positive environmental cultures (both MRSA and Gram-negative bacilli).

\begin{tabular}{|c|c|c|c|c|c|}
\hline & $\begin{array}{r}\text { Positive culture } \\
\text { Freq (\%) }\end{array}$ & $\begin{array}{r}\text { Negative culture } \\
\text { Freq }(\%)\end{array}$ & $\begin{array}{r}\text { Total } \\
\text { freq }(\%)\end{array}$ & $\begin{array}{r}\text { Odds Ratio } \\
(\mathrm{OR})(95 \% \mathrm{Cl})\end{array}$ & $p$-value \\
\hline $\begin{array}{l}\text { Treated } \\
\text { with TiO2 }\end{array}$ & $6(4.4 \%)$ & $129(95.6 \%)$ & $135(100 \%)$ & \multirow{2}{*}{$2.95(1.25-6.94)$} & \multirow{2}{*}{$p=0.01$} \\
\hline $\begin{array}{l}\text { Untreated } \\
\text { with } \mathrm{TiO} 2\end{array}$ & $68(12.1 \%)$ & 495 (87.9\%) & $563(100 \%)$ & & \\
\hline Ad hoc sampling & $66(14.1 \%)$ & $403(85.9 \%)$ & $469(100 \%)$ & \multirow{2}{*}{$4.52(2.132-9.615)$} & \multirow{2}{*}{$p<0.0001$} \\
\hline Planned sampling & $8(3.5 \%)$ & $221(96.5 \%)$ & $229(100 \%)$ & & \\
\hline
\end{tabular}




\section{Table II. Distribution of positive environment cultures (both MRSA and Gram-negative bacilli) among various culture sites}

\begin{tabular}{lrr} 
Culture site & No. of positive cultures & Total no. of samples \\
\hline Common blood pressure cuff* & $20(20.0 \%)$ & 100 \\
\hline Screening curtain around the bed* & $14(23.7 \%)$ & 59 \\
\hline Bedside locker - drawer & $6(15.0 \%)$ & 40 \\
\hline Bedside locker - top surface & $5(12.2 \%)$ & 41 \\
\hline Carpenter ruler* & $5(10.2 \%)$ & 49 \\
\hline Pull switch for wall-mount lamp & $7(11.9 \%)$ & 59 \\
\hline Door handle & $5(10.0 \%)$ & 50 \\
\hline Main light switch & $4(4.0 \%)$ & 100 \\
\hline Hemodynamic monitor screen & $4(8.0 \%)$ & 50 \\
\hline Tap at basin & $3(3.0 \%)$ & 100 \\
\hline Door pad & $1(2.0 \%)$ & 50 \\
\hline
\end{tabular}

*Surfaces were not treated with $\mathrm{TiO}_{2}$ coating.

Overall $\mathrm{TiO}_{2}$ efficacy in preventing environmental contamination was $63.6 \%$ and there was no suggestion of significantly reduced $\mathrm{TiO} 2$ efficacy over time. However, $\mathrm{TiO} 2$ efficacy was only $17.8 \%$ in sub-group analysis of environmental samples from MRSA exposed environment obtained during ad hoc sampling. Bivariate analysis also showed that $\mathrm{TiO} 2$ treatment did not confer prevention against environmental contamination in MRSA environment $(p=0.66)$.
Factors influencing environmental contamination

After adjusting for confounding variables such as sampling schedule, sampled sites, with or without $\mathrm{TiO}_{2}$ treatmentin multiple logistic regression, ad hoc sampling in MRSA environment was the only independent factor influencing positive culture results (OR 4.47, $95 \% \mathrm{Cl} 2.03$ - 9.82, $\mathrm{p}<0.001)$. Treatment with $\mathrm{TiO}_{2}$ was no longer a predictor for positive culture results.

Table III. Distribution of positive environmental cultures from treated versus untreated surfaces in MRSA versus non-MRSA environment

\begin{tabular}{|c|c|c|c|c|c|}
\hline & & $\begin{array}{l}\text { Culture } \\
\text { positive } \\
\text { Freq (\%) }\end{array}$ & $\begin{array}{l}\text { Culture } \\
\text { negative } \\
\text { Freq (\%) }\end{array}$ & $\begin{array}{r}\text { Total Freq } \\
(\%)\end{array}$ & $\begin{array}{r}p \text {-value } \\
\text { (Chi Square } \\
\text { Test) }\end{array}$ \\
\hline \multirow{2}{*}{$\begin{array}{l}\text { MRSA environment } \\
\text { (Ad hoc sampling) }\end{array}$} & Treated surface & $6(12.0 \%)$ & $44(88.0 \%)$ & $50(100 \%)$ & \multirow[b]{2}{*}{0.621} \\
\hline & Untreated surface & $62(14.6 \%)$ & $\begin{array}{r}363 \\
(85.4 \%) \\
\end{array}$ & $425(100 \%)$ & \\
\hline \multirow{2}{*}{$\begin{array}{l}\text { Non-MRSA environment } \\
\text { (Planned sampling) }\end{array}$} & Treated surface & $0(0.0 \%)$ & $85(100 \%)$ & $85(100 \%)$ & \multirow[b]{2}{*}{0.051} \\
\hline & Untreated surface & $6(4.3 \%)$ & $\begin{array}{r}132 \\
(95.7 \%)\end{array}$ & $138(100 \%)$ & \\
\hline
\end{tabular}




\section{Discussion}

We examined the efficacy of titanium dioxide $\left(\mathrm{TiO}_{2}\right)$ coating, a photocatalytic disinfectant, as an adjunct to conventional terminal cleaning in reducing hospital environmental contamination. $\mathrm{TiO}_{2}$ efficacy in preventing contamination in an environment exposed to MRSA patients was only $17.8 \%$. Multivariate analysis suggested that exposure of the environment to MRSA patients was the only independent factor influencing positive cultures. Prevalence of MRSA was the highest for the common blood pressure cuff and screening curtain.

$\mathrm{TiO}_{2}$ substrates with photocatalytic properties act as disinfectants. ${ }^{15}$ Newer photocatalytic compounds can be activated with visible light and are efficacious against organisms such as MRSA. 16,17 However, research so far has been limited to in vitro studies.

In the real hospital environment, we found $\mathrm{TiO}_{2}$ to be highly efficacious in preventing environmental contamination in a non-MRSA exposed environment. None of the samples from treated surfaces that were not exposed to MRSA returned positive during planned sampling. This was consistent with previous in vitro studies where $\mathrm{TiO}_{2}$ in combination with other metals such as copper and silver was found to have antibacterial properties against Staphylococcus aureus and MRSA. ${ }^{18,19}$

However, $\mathrm{TiO}_{2}$ efficacy notably decreased to only $17 \%$ in sub-group analysis of samples taken from MRSA exposed environment and did not significantly confer prevention against overall environmental recontamination. Treatment with $\mathrm{TiO}_{2}$ was also not an independent factor influencing positive cultures. This could have been due to the chemical composition of the $\mathrm{TiO}_{2}$ compound in this particular coating. In an in vitro study by Necula et al., $\mathrm{TiO}_{2}$ combined with silver nanoparticles was $100 \%$ efficacious in eliminating MRSA. In oxidized titanium without silver nanoparticles, a 1000 fold increase in colony forming units was observed instead. ${ }^{19}$ Our study agent did not contain silver nanoparticles.

MRSA exposure was the only independent factor influencing positive cultures in multiple logistic regression analysis (OR 4.47). This can be attributable to the ability of MRSA to contaminate a large variety of hospital items and withstand desiccation; surviving in hospital dust for up to a year. ${ }^{13}$ Rapid environmental recontamination with MRSA was also observed by Hardy et al despite decontamination with hydrogen peroxide vapor. MRSA was also isolated from $17.2 \%$ of sampled sites after conventional decontamination methods. $^{20}$

Overall prevalence of environmental contamination over 24 months was $11 \%$. However, environmental contamination was higher for untreated surfaces (12.1\% versus $4.4 \%$ for treated surfaces) and ad hoc samples from MRSA environment $(14.1 \%$ versus $3.5 \%$ for planned sampling from non-MRSA environment). Dancer et al. reported MRSA being present in 5.8\% of samples taken from near patient sites such as bed hoist, over bed table and locker, and 1.9\% from sites further from patients such as door handle, blood pressure pump stand and computer keyboard at the nurses station. ${ }^{11}$ This was similar to what we observed in a non-MRSA environment.

Of the sites sampled, the common blood pressure cuff and the screening curtain were more likely to be MRSA positive. Dancer et al. concluded in two separate studies, that near patient sites were significantly more likely to be contaminated with meticillin sensitive Staphylococcus aureus (MSSA)/MRSA. MSSA and MRSA were categorized together for analysis because of their small sample size. The sites identified were the bed frame, bedside locker and overbed table. ${ }^{11,12}$ These three sites were similarly sampled in our study, but were not significantly more contaminated than the other sampled sites. The increased likelihood of contamination of the common blood pressure cuff and screening curtain could have been a result of direct physical contact with the patient for the former and frequent contact with possibly contaminated hands of the patient and healthcare workers. This was in addition to the less frequent cleaning of these surfaces. Blood pressure cuffs were used for each patient for each episode of admission and screening curtains were laundered upon discharge of the patient.

There were limitations to our study. The number of environmental samples from treated surfaces were smaller than that from untreated surfaces as a result 
of more MRSA patients being allocated to untreated rooms during ad hoc sampling. This could have limited the assessment of overall efficacy of $\mathrm{TiO}_{2}$ treated surfaces. Treated surfaces were also not re-applied during the study period. $\mathrm{TiO}_{2}$ efficacy could have been reduced as a result of wear-and-tear of the 'binder' in the coating on these frequently touched surfaces. Pulse-field gel electrophoresis was not performed on patient and environmental cultures. We were thus unable to conclude the extent of environmental MRSA contamination attributable to MRSA patients residing in the vicinity of the environmental sampling. We were also unable to quantify the efficacy of $\mathrm{TiO}_{2}$ as we had opted for a qualitative approach with the use of environmental swabs. However, as this study was designed to examine the efficacy of $\mathrm{TiO}_{2}$ over 24 months instead of evaluating its efficacy in reduction in environmental contamination immediately post treatment, the information obtained through environmental swabs would still provide useful information on the efficacy of $\mathrm{TiO} 2$ in the real hospital setting. There was a very low prevalence of GNB among the environmental samples and this limited the findings of our study to that of mainly MRSA.

Given the potential of $\mathrm{TiO}_{2}$ to reduce environmental contamination and hence hospital acquired infections, further research could be conducted to evaluate the factors that influence the efficacy of $\mathrm{TiO}_{2}$ against MRSA in the real world setting particularly using randomized study designs. The use of $\mathrm{TiO} 2$ could potentially reduce the presence of environmental contamination which could arise from varying standards of environmental cleaning. $\mathrm{TiO} 2$ would complement current environmental cleaning practices.

We found environmental exposure to MRSA to be the only independent factor that influenced positive environmental cultures. Photocatalytic titanium dioxide did not appear to confer protection against environmental contamination, especially when sampling from an environment exposed to MRSA. More research could be done in identifying and evaluating innovative strategies in reducing environmental contamination by particularly MRSA.

\section{Acknowledgements}

We would like to thank the support of the nurses from Infection Control, Singapore General Hospital, Singapore who have kindly assisted in conducting the environmental sampling for the study and for kindly providing information on the cleaning protocols.

This project was funded by a research grant (SHF/07/ BOT(2)/007(R)-3) by SingHealth Foundation.

\section{Previous Presentation}

The preliminary findings of this study were previously presented as a poster at SingHealth Duke-NUS Scientific Congress 2010.

\section{References}

1. Klein E, Smith DL, Laxminarayan R. Hospitalisations and death caused by methicillin resistant Staphylococcus aureus, United States, 1999 - 2005. Emerging Infectious Diseases 2007; 13(12): 1840-1846. http://dx.doi.org/10.3201/ eid1312.070629

2. ECDC. Antimicrobial resistance surveillance in Europe 2010. Annual report of the european antimicrobial resistance surveillance network (EARS-Net). http://www.ecdc.europa.eu/ en/publications/Publications/1111_SUR_AMR_data.pdf.pdf

3. Dulon M, Haamann F, Peters C, Schalbon A, Nienhaus A. MRSA prevalence in European healthcare settings: a review. BMC Infectious Diseases 2011; 11: 138. http://dx.doi. org/10.1186/1471-2334-11-138

4. Hsu LY, Tan TY, Joreen R, et al. Antimicrobial Drug Resistance in Singapore Hospital. Emerging Infectious Diseases 2007; 13(12): 1944-1947. http://dx.doi.org/10.3201/ eid1312.070299

5. Mera RM, Suaya JA, Amrine-Madsen H, et al. Increasing role of Staphylococcus aureus and community-acquired methicillinresistant Staphylococcus aureus infections in the United States: a 10-year trend of replacement and expansion. Microb Drug Resist 2011; 17(2): 321-328. http://dx.doi.org/10.1089/ mdr.2010.0193

6. Centres for Disease Control and Prevention. Hospital acquired infections. The burden. http://www.cdc.gov/HAl/burden.html. Accessed 31 July 2012.

7. Devlin K. Every MRSA case costs NHS an extra $£ 9,000$. The Telegraph. 25 June 2008. http://www.telegraph.co.uk/ news/uknews/2194132/Every-MRSA-case-costs-NHS-anextra-9000.html. Accessed 31 July 2012.

8. Hardy KJ, Oppenheim BA, Gossain S, Gao F, Hawkey PM. A study of the relationship between environmental contamination with methicillin-resistant Staphylococcus aureus (MRSA) and patients' acquisition of MRSA. Infect Control Hosp Epidemiol 2006; 27: 127-132. http://dx.doi.org/10.1086/500622

9. Dancer SJ. The role of environmental cleaning in the control of hospital-acquired infection. J Hosp Infect 2009: 73; 378-385. http://dx.doi.org/10.1016/j.jhin.2009.03.030

10. Boyce JM. Environmental contamination makes an important contribution to hospital infection. J Hosp Infect 2007; 65(52): 50-54. http://dx.doi.org/10.1016/S0195-6701(07)60015-2 
11. Dancer SJ, White L, Robertson C. Monitoring environmental cleanliness on two surgical wards. International Journal of Environmental Health Research 2008; 18(5): 357-364. http:// dx.doi.org/10.1080/09603120802102465

12. Dancer SJ, White LF, Lamb J, Girvan EK, Robertson K. Measuring the effect of enhanced cleaning in a UK hospital: a prospective cross-over study. BMC Medicine 2009; 7: 28. http://dx.doi.org/10.1186/1741-7015-7-28

13. WagenvoortJH, Sluijsmans W, Penders RJ. Better environmental survival of outbreak vs. sporadic MRSA isolates. J Hosp Infect 2000; 45: 231-234. http://dx.doi.org/10.1053/jhin.2000.0757

14. Singapore General Hospital. Quality Measures. http://www. sgh.com.sg/patient-services/our-commitment-to-patients/ pages/quality-measures.aspx. Accessed 31 July 2012.

15. Maness PC, Smolinski S, Blake DM, Huang Z, Wolfrum EJ, Jacoby WA. Bactericidal activity of photocatalytic $\mathrm{TiO} 2$ reaction: toward an understanding of its killing mechanism. Appl Environ Microbiol 1999; 65(9): 4094-4098.

16. Wong MS, Chu WC, Sun DS, et al. Visible light-induced bactericidal activity of nitrogen-doped titania photocatalyst in eliminating the human pathogens. Appl Environ Microbiol 2006; 72: 6111-6116. http://dx.doi.org/10.1128/AEM.0258005
17. Cheng CL, Sun DS, Chu WC, et al. The effects of the bacterial interaction with visible-light responsive titania photocatalyst on the bactericidal performance. Journal of Biomedical Science 2009; 16: 7. http://dx.doi.org/10.1186/1423-012716-7

18. Haenle M, Fritsche A, Zietz C, et al. An extended spectrum bactericidal titanium dioxide (TiO2) coating for metallic implants: in vitro effectiveness against MRSA and mechanical properties. I Mater Sci Mater Med 2011; 22(2): 381-387. http://dx.doi.org/10.1007/s10856-010-4204-4

19. Necula BS, Fratila-Apachitei LE, Zaat SA, Apachitei I, Duszczyk J. In vitro antibacterial activity of porous TiO2-Ag composite layers against methicillin-resistant Staphylococcus aureus. Acta Biomater 2009; 5(9): 3573-3580. http://dx.doi. org/10.1016/j.actbio.2009.05.010

20. Hardy KJ, Gossain S, Henderson N, et al. Rapid recontamination with MRSA of the environment of an intensive care unit after decontamination with hydrogen peroxide vapour. I Hosp Infect 2007; 66: 360-368. http://dx.doi.org/10.1016/j. jhin.2007.05.009 